\title{
Quantitative Evaluation in Estimating Sources Underlying Brain Oscillations Using Current Source Density Methods and Beamformer Approaches
}

\author{
Tamesh Halder, ${ }^{1}{ }^{*}$ Siddharth Talwar, ${ }^{1 *}$ Amit Kumar Jaiswal, ${ }^{1}$ and ${ }^{(D)}$ Arpan Banerjee ${ }^{1}$
}

https://doi.org/10.1523/ENEURO.0170-19.2019

${ }^{1}$ Cognitive Brain Dynamics Lab, National Brain Research Centre, NH8, Manesar, Haryana 122052, India

\begin{abstract}
Brain oscillations from EEG and MEG shed light on neurophysiological mechanisms of human behavior. However, to extract information on cortical processing, researchers have to rely on source localization methods that can be very broadly classified into current density estimates such as exact low-resolution brain electromagnetic tomography (eLORETA), minimum norm estimates (MNE), and beamformers such as dynamic imaging of coherent sources (DICS) and linearly constrained minimum variance (LCMV). These algorithms produce a distributed map of brain activity underlying sustained and transient responses during neuroimaging studies of behavior. On the other hand, there are very few comparative analyses that evaluates the "ground truth detection" capabilities of these methods. The current article evaluates the reliability in estimation of sources of spectral event generators in the cortex using a two-pronged approach. First, simulated EEG data with point dipoles and distributed dipoles are used to validate the accuracy and sensitivity of each one of these methods of source localization. The abilities of the techniques were tested by comparing the localization error, focal width, false positive (FP) ratios while detecting already known location of neural activity generators under varying signal-to-noise ratios (SNRs). Second, empirical EEG data during auditory steady state responses (ASSRs) in human participants were used to compare the distributed nature of source localization. All methods were successful in recovery of point sources in favorable signal to noise scenarios and could achieve high hit rates if FPs are ignored. Interestingly, focal activation map is generated by LCMV and DICS when compared to eLORETA while control of FPs is much superior in eLORETA. Subsequently drawbacks and strengths of each method are highlighted with a detailed discussion on how to choose a technique based on empirical requirements.
\end{abstract}

Key words: DICS; EEG; eLORETA; LCMV; MEG; MNE

\section{Significance Statement}

State-of-the-art methods of source localization techniques, e.g., current density methods, minimum norm estimates (MNE) and beamformers report distributed brain activity patterns that are often not in consensus for a putative task. This article offers ground truth validation of these techniques in the context of different kind of source detections, e.g., determining the sources underlying key events (evoked potentials) and steady state brain oscillations (band limited brain activity). The broader goal is to help cognitive neuroscientists select the most effective source localization technique that is in sync with the signal processing needs required for targeting a specific question. 


\section{Introduction}

Cortical oscillations play an important role in governing basic cognitive functions (Edelman and Mountcastle, 1978; Bressler and Kelso, 2001; Buzsáki and Draguhn, 2004). Several researchers have suggested that electromagnetic brain activity at specific frequency bands carries meaningful information about neural function, e.g., alpha waves at $10 \mathrm{~Hz}$ (Llinás et al., 1999; Bollimunta et al., 2008), beta at 15-30 Hz (Brovelli et al., 2004), and gamma at $30 \mathrm{~Hz}$ and above (Bressler et al., 1993; Varela et al., 2001; Cheyne and Ferrari, 2013). Concurrently, timelocked transient responses have been useful for decades in electrophysiological research, both for understanding basic neurobiological functions as well as in clinical and other applications (Picton et al., 1974; Kutas et al., 1977; Clark et al., 1994; Pantev et al., 1995; Cheyne et al., 2006). Hence, identifying the neural generators of sustained cortical oscillations and task-specific transient neural responses from EEG/MEG is an extensive topic of research. Once identified with adequate reliability, the focal localization of sources will eventually reveal the underlying large-scale network governing cognitive tasks.

There are several source localization methods in the literature, commonly known under the umbrella of inverse methods (Hämäläinen and Sarvas, 1989). Most of these techniques are based on fitting single/multiple dipolar cortical source/sources within a defined cortical volume based on some assumptions about relationships between the sources (Hämäläinen and IImoniemi, 1994; Van Veen et al., 1997; Ishii et al., 1999; Gross et al., 2001; Liu et al., 2002; Hillebrand and Barnes, 2003; Sato et al., 2004). Some methods consider sources to have minimum correlation, e.g., synthetic aperture magnetometry (SAM; Hillebrand and Barnes, 2003), linearly constrained minimum variance (LCMV) spatial filtering (Van Veen et al., 1997; Murzin et al., 2011). There are specialized measures that detect generators of oscillatory brain signals by considering maximum coherence between prospective sources, e.g., dynamic imaging of coherent sources (DICS; Gross et al., 2001) and entropy based metrics (Lina et al., 2014). DICS is a frequency domain extension of beamforming methods over the initially developed time-domain beamformers, e.g., LCMV and SAM (Ishii et al., 1999), which are primarily used for determining the sources underlying time-locked event-related potentials/event-related fields

Received May 8, 2019; accepted June 25, 2019; First published July 16, 2019. The authors declare no competing financial interests.

Author contributions: T.H., S.T., and A.B. designed research; T.H., S.T., and A.B. performed research; T.H., S.T., and A.B. analyzed data; T.H., S.T., and A.B. wrote the paper; A.K.J. and A.B. contributed unpublished reagents/ analytic tools.

This work was supported by Department of Biotechnology, Government of India Grants BT/RLF/Reentry/31/2011 and BT/07/IYBA/2013 (to A.B.)

${ }^{*}$ T.H. and S.T. contributed equally to this work.

Correspondence should be addressed to Arpan Banerjee at arpan.nbrc@ gov.in or banerjee2007@gmail.com.

https://doi.org/10.1523/ENEURO.0170-19.2019

Copyright @ 2019 Halder et al.

This is an open-access article distributed under the terms of the Creative Commons Attribution 4.0 International license, which permits unrestricted use, distribution and reproduction in any medium provided that the original work is properly attributed.
(ERPs/ERFs) components. In EEG, where deeper sources can affect scalp potentials, current density techniques such as minimum norm estimates (MNE; Hämäläinen and Ilmoniemi, 1994) and exact low-resolution brain electromagnetic tomography (eLORETA; Pascual-Marqui, 2007) have been the method of choice. Although dynamic statistical parametric mapping (dSPM; Liu et al., 2002) and sparse Bayesian learning (SBL; Ramírez et al., 2010) have been developed to improve on the estimates of spatial filter detection, eLORETA is still by far one of the most robust methods for EEG source localization. eLORETA directly estimates current source density, a biophysically relevant parameter over a grid of plausible cortical locations for both detection of time-locked activity, e.g., in ERP/ERF or frequency-locked activity, e.g., spontaneous frequency bursts or steady state oscillatory responses to periodic stimuli. Nonetheless, the source estimated by all methods is broadly influenced by depth, signal-to-noise strength of the neural activity, as well as the correlation in the covariance of the signals (Belardinelli et al., 2012) and redundant informational content of high temporal resolution data. Often, these manifest in distributed source activity estimation with diminished statistical power

The accuracy of the location of neural activity along with lower false positives (FPs) should be the expectation from any source localization technique. In this article, we evaluate the performance of the key current density techniques: eLORETA and MNE, and beamformer approaches: DICS and LCMV, on simulated EEG data. Since LCMV and DICS belong to the same class of beamformers, they were compared against eLORETA, to quantify localization efficiency between beamformers versus current density measures. We compared the results from eLORETA and DICS on a paradigm of evoked $40-\mathrm{Hz}$ auditory steady state responses (ASSRs) and eLORETA, MNE, and LCMV for detecting the source of N100 activity when the same data were epoched time locked to the stimulus onset. Many inverse methods can localize a transient or steady state response or both. However, the biological relevance or interpretation of these different information processing events can be very distinct. There are comparison studies that evaluate the performance across different methods (Bradley et al., 2016; Hedrich et al., 2017) or sometimes the performance of detecting a focal cortical source across modalities EEG and MEG (Srinivasan et al., 2006; Mideksa et al., 2015). In this article, our focus was to compare the specificity and sensitivity of some of the prominent algorithms primarily chosen based on their conceptual difference current density estimate versus beamforming to provide a basis for choosing one above the other when faced with the issues of transient or steady state response. Very rigorous comparison metrics, e.g., localization error, spatial spread, and FP percentage, were used to evaluate accuracy and sensitivity of results along with an evaluation of the performance of these methods at different depths of dipole placement in the simulated EEG data. Subsequently, empirical EEG data during N100 and ASSRs were used to draw comparisons among distributed nature of source activity patterns generated by these methods. 


\section{Materials and Methods}

\section{Generation of synthetic EEG data}

To localize the oscillatory activity, as well as the transient response, we simulated a time-varying sinusoid at $40 \mathrm{~Hz}$ and a mixture of Gaussian pulses, respectively. Both generated signals were free of noise and were consequently added to the acquired empirical baseline, for realistic noise simulation. The magnitude of the dipolar source dynamics in cortical locations are represented by

$$
q_{i}(t)=\sin (2 \pi 40 t)+\epsilon(0, \sigma),
$$

where $q_{i}(t)$ is the electric dipole moment at location $i$ and at time $t, \epsilon$ is white noise with zero mean and SD $\sigma$. We compared three conditions with respect to number of sources, by placing single-point dipole, two-points dipoles, and distributed dipoles in a Montreal Neurologic Institute (MNI) brain template according to the MNI (Müller and Weisz, 2012). Single-point source was placed at around the superior temporal region, in the left hemisphere [MNI coordinates: $(-60,-28,6)]$. Two sources were placed, one in the left hemisphere [MNI coordinates: $(-60,-28,6)]$ and the other in the right hemisphere [MNI coordinates: $(64,-24,6)]$ around the superior temporal region. Approximately, 100-point sources were placed within a spherical volume with radius of $12 \mathrm{~mm}$ in the left hemisphere centered around the superior temporal region at $(-60,-28,6)$, according to brain template. Another set of 100-point sources was placed around the right hemisphere auditory cortex seed area at $(64,-24,6)$, defining the distributed source condition. The resolution of the grid chosen for dipole simulation was $5 \mathrm{~mm}$, and ft_prepare_leadfield.m code of FieldTrip toolbox was used for this purpose. Dipole moment orientations were assumed to be along the radial direction with respect to the BEM surface, to retain simplicity. We computed the scalp potentials for EEG at realistic sensor locations by applying a forward model (Mosher et al., 1999; Baillet et al., 2001) with realistic headshape using $\mathrm{ft}$ _dipolesimulation.m of the FieldTrip toolbox.

$$
v_{r}(t)=\mathbf{L}^{T}\left(r, \mathbf{r}_{q}\right) \cdot q(t)
$$

where $v$ is the electric potential at sensor location $r, r_{q}$ represents all source locations, $\mathbf{L}$ represents the "lead field kernel," $(.)^{T}$ represents transpose, and $q(t)$ is the dipole moment. Synthetic EEG data were generated by varying signal-to-noise ratio (SNR) at the source space. Physiologic SNR was estimated using a statistical measure, $10 \log _{10}\left[s / \sigma_{b}\right]$, where $s$ is peak-to-peak amplitude of EEG data during rhythmic auditory stimulation (see experimental methods below), and $\sigma_{b}$ is the SD of the baseline data. We chose a wide range of SNRs $(19,22,25$, $28,31 \mathrm{~dB}$ ) to simulate mixture of Gaussian pulses mimicking transient response, both above and below the estimated physiologic SNR level (25 dB), to allow us to evaluate the sensitivity of each method. Further, we introduced time lags between the signals generated from the left and right hemispheres for two and distributed dipole models. Time delays of $0,15,30,45 \mathrm{~ms}$ were added to the
Gaussian pulse generated from the right hemisphere. Figure 1 shows simulated EEG activity on scalp surface with bilateral auditory cortical sources. Following Goldenholz et al. (2009), SNR was computed in dB using the following equation:

$$
S N R_{d B}=10 \log _{10}\left[\frac{1}{U} \sum_{u=1}^{U} \frac{v_{u}^{2}}{\eta_{u}^{2}}\right]
$$

where $U$ is total sensor count, and $v$ is the signal on sensor $u \in(1,2, \cdots U)$ provided by the forward model for a source with unit amplitude. The sensor space variance is expressed as $\eta_{u}^{2}=\sigma^{2}\left(\mathbf{L L}^{\top}\right)_{u}$. Subsequently, for each time lag scenario, we simulated the Gaussian signal of five SNR values. Additionally, we also simulated sinusoidal signals mimicking oscillatory activity with different values of power spectra at $40 \mathrm{~Hz}$. The power values were chosen with respect to the power spectrum computed for the empirical binaural condition data, such that the power of simulated sinusoidal at $40 \mathrm{~Hz}$ was $50 \%, 75 \%, 100 \%$, $125 \%$, and $150 \%$ of the power at $40 \mathrm{~Hz}$ of the binaural condition, illustrated in Figure 1. Further, phase lags were introduced between the signals generated in left and right hemisphere: $0, \pi / 2, \pi$, and $3 \pi / 2$. Therefore, all the power ratio scenarios were computed for each phase lag condition.

\section{Source localization methods}

The basic goal of any source localization technique is to compute the dipolar source locations and strengths inside the brain from measurements on the scalp (inverse of Equation 2). In other words, the objective is to estimate the spatial filter $\mathbf{W}_{S}$ from the relation

$$
q(t)=\mathbf{W}_{S} \cdot \mathbf{V}(t)
$$

where $q(t)$ is the dipole moment at time $t, \mathbf{W}_{S}$ is the spatial filter matrix, and $\mathbf{V}$ is vector representation of all sensor time series. Obviously, the system of equations represented by Equation 4 is ill-posed, as the number of sensors (dimension of vector $\mathbf{V}$ ) is finite, but the number of dipoles is unknown. Thus, different source localization methods attempt to estimate the $\mathbf{W}_{S}$ using diverse constraints posed by anatomy of the brain and functional relationships among brain areas during ongoing task.

\section{LCMV}

LCMV belongs to the class of "beamformer" methods that enhances a desired signal while suppressing noise and interference at the output array of sensors (Barnes and Hillebrand, 2003). LCMV is built on an adaptive spatial filter whose weights are calculated using covariance matrix of EEG/MEG time series data. A spatial filter computes the variance of the total source power which is allowed to vary but the output of the filtered lead field is kept constant. As a result, the beamformer output is 
A

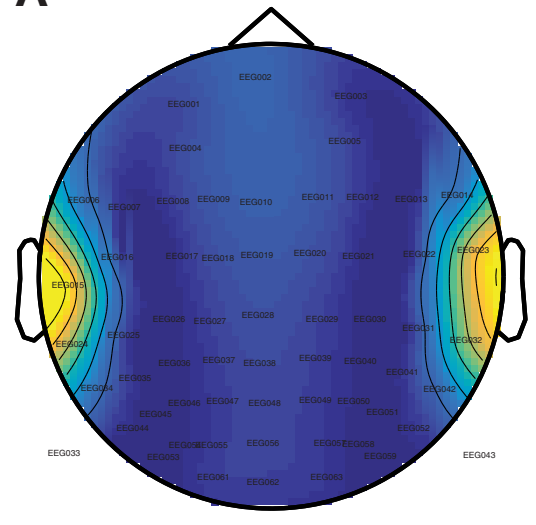

$\begin{array}{lllllll}3 & 2.5 & 2 & 1.5 & 1 & 0.5 & 0\end{array}$
Power spectral Density for different signal levels

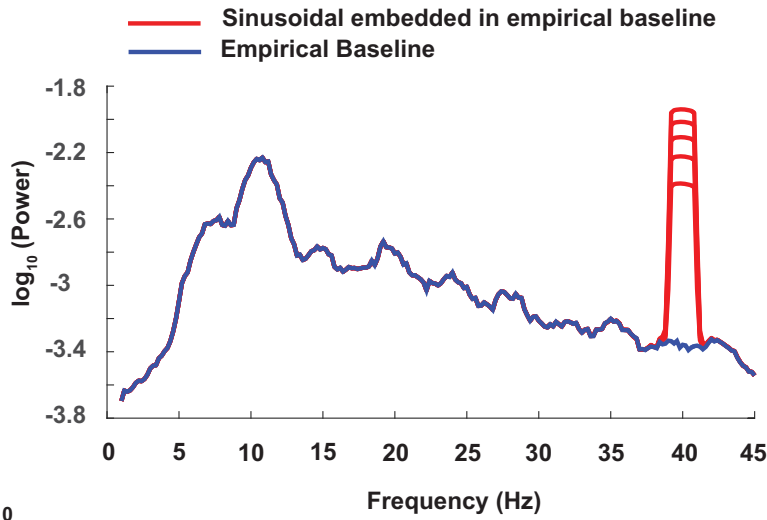

$x 10$

Relative Change in Power

B

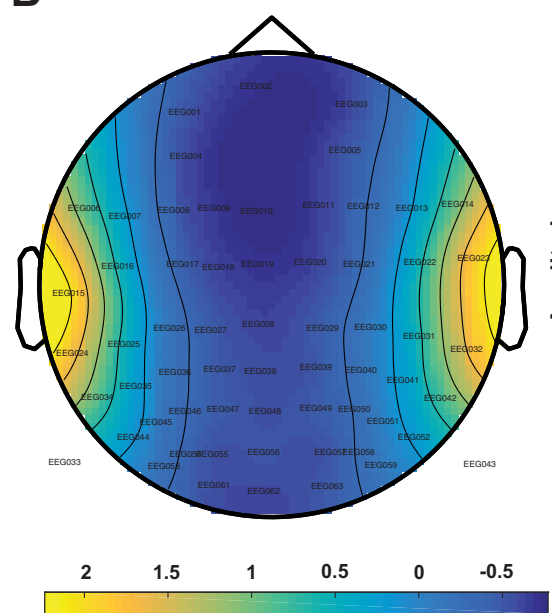

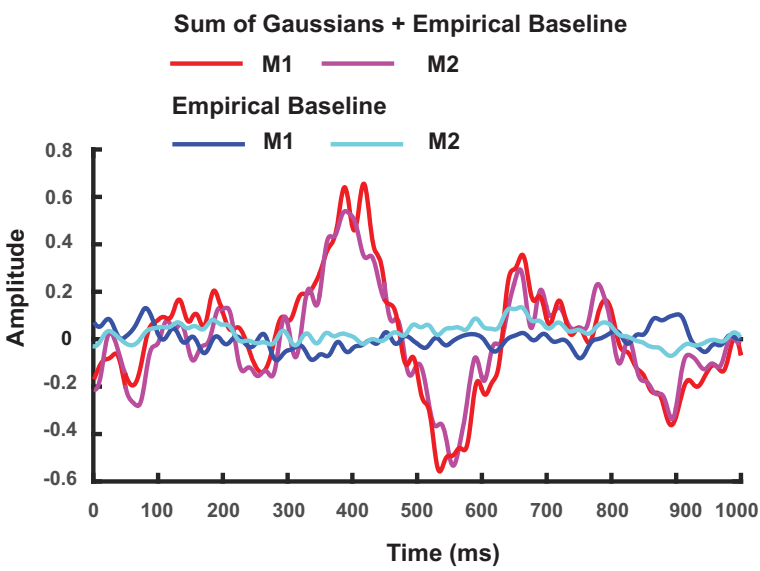

Sum of Gaussians + Empirical Baseline

M1 M2

Relative Change in Power

Figure 1. A, Topoplot of the difference in peak of spectral power at $40 \mathrm{~Hz}$ obtained from simulated EEG data where dipolar source time series was represented by a sinusoidal signal with frequency $40 \mathrm{~Hz}$ embedded in empirical resting state EEG and empirical resting state EEG as baseline. Spatially averaged power spectra obtained from averaging the channel-by-channel spectrum from hypothetical scalp sensors are plotted in logarithmic scale. The time series on the scalp were obtained by applying forward modeling techniques on dipolar sources at auditory cortex locations using the Boundary element method (BEM). B, Topoplot of the peak of difference signal when a mixture of Gaussian pulses was used to simulate ERP and empirical resting state EEG as baseline in BEM model as described in $\boldsymbol{A}$. The time series for dipole dynamics are plotted at hypothetical M1 and M2 sensors located near to the auditory cortices. The positive peak at $400 \mathrm{~ms}$ was used for generating the topoplot.

maximized for the target source but other source contributions are suppressed. LCMV attempts to minimize the beamformer output power

$$
\mathbf{P}=\mathbf{W}_{S}^{\top} \mathbf{C} \mathbf{W}_{S} \mathrm{~S}
$$

where $\mathbf{C}$ is the data covariance matrix. The entries to spatial filter matrix can be expressed as

$$
\mathbf{W}_{S}=\frac{\mathbf{L}^{\top} \mathbf{C}^{-1}}{\mathbf{L}^{\top} \mathbf{C}^{-1} \mathbf{L}}
$$

where $\mathbf{L}$ is lead field matrix, and the following constraint is maintained $\mathbf{W}_{S} \cdot \mathbf{L}^{T}=1$.

\section{DICS}

DICS beamformer (Gross et al., 2001) works with same constraint assumption of LCMV but extends the computation of spatial filter to the frequency domain. Here, sensor level cross-spectral density (CSD) matrix replaces the covariance matrix, and the spatial filter is applied to sensor level CSD to reconstruct the source level CSD of all combination of pairwise voxels. Hence, DICS directly estimates the interaction between sources at respective frequencies. The weight function can be written as

$$
\mathbf{W}_{S}(f)=\frac{\mathbf{L}^{\top} \mathbf{C}^{-1}(f)}{\mathbf{L}^{T} \mathbf{C}^{-1}(f) \mathbf{L}} .
$$




\section{eLORETA}

eLORETA (Pascual-Marqui, 2007) combines the leadfield normalization with the 3D Laplacian operator under the constraint of smoothly distributed sources. Compared to DICS and LCMV, where the constraint equation $\mathbf{W}_{S} \cdot \mathbf{L}^{T}=1$ is used, eLORETA seeks to minimize the product $\mathbf{H}=\mathbf{W}_{S} \mathbf{L}^{T}$.

$$
\mathbf{W}_{S}=\left[\mathbf{L}^{\top}\left(\mathbf{L} \mathbf{C}^{-1} \mathbf{L}^{T}+\mathbf{a H}\right)^{\dagger} \mathbf{L}\right]^{1 / 2},
$$

where $a$ is regularization parameter, and $\dagger$ is the MoorePenrose pseudo-inverse, which is equal to the common inverse if the matrix is non-singular. $\mathbf{H}$ is also called the centering matrix or the surface Laplacian. Low-resolution imaging results in weak performance for recovering of multiple sources when the point-spread functions of sources overlap. Other methods have also tried to combine surface Laplacian with LCMV (Murzin et al., 2013), to estimate source-level connectivity.

\section{MNE}

MNE has been a popular choice to localize evoked activity and tracking the distribution of the activations over a period of time. MNE is a distributed inverse solution that discretizes the source space into locations on the cortical surface or in the brain volume using a large number of equivalent current dipoles. It estimates the amplitude of all modeled source locations simultaneously and recovers a source distribution with minimum overall energy that produces observed sensor data consistent with the measurement (Hämäläinen and Ilmoniemi, 1994; Ou et al., 2009). The current density $q$ can be calculated as

$$
\left.\mathbf{q}=\left[\mathbf{C L}^{T}\left(\mathbf{L} \mathbf{C} \mathbf{C}^{T}+\lambda \mathbf{C}\right)^{-1}\right)\right] .
$$

\section{Measurements used for face validity of inverse algorithms}

Using simulated data to test a method provides mechanism for ground truth validation. The exact location of a putative dipolar source is elusive in nature for real data, however, one can certainly set-up simulations when performance of a particular method needs evaluation. We employed three complementary measures to provide face-validity of the eLORETA in comparison with LCMV and DICS.

\section{Localization error estimation}

Inverse methods estimate a cluster of point dipolar sources. To measure how much error is involved in source localization, we first computed the z-scores for all voxels. Further, we thresholded the z-scores at 99.99th percentile and identified the cluster closest to the dipole location. Then we measured the Euclidean distance between the voxel with the maximum z-score of the nearest cluster source points and the actual source/dipole coordinates, to give us the localization error. This was done for each hemisphere, separately. Consequently, the net localization errors are computed by summing up across two hemispheres and compared for eLORETA, MNE, LCMV, and DICS.

\section{Degree of focal localization}

The size of a cluster in terms of sum of distances of all points from the voxel with the maximum z-score, gives a measure of focal localization of sources. After finding the $z$-scores of all voxels, we thresholded the scores at 99.99th percentile and identified the cluster of source points closest to the dipolar location. Further, we computed the total sum of distances of each voxel in the nearest cluster, from the voxel in the cluster with the maximum z-score, as a measure of the spatial distribution of the estimated source. This gave us a quantitative approach to evaluate the degree of focal source localization. For practical reasons, cluster width computation was done for each hemisphere separately (Murzin et al., 2013).

\section{Performance evaluation at various depths}

The localization of deep sources has been the main factor limiting detection of true sources from MEG/EEG data. This is important, since deep cortical areas constitute $30 \%$ of the cortical sources (Hillebrand and Barnes, 2002). We studied the effects of depth by positioning the dipoles at different distances from the auditory cortical locations mentioned earlier. The depth was varied along the $x$-axis, from 0 to $20 \mathrm{~mm}$ in steps of $1 \mathrm{~mm}$, towards the center of the brain, in both hemispheres. Further, we computed the localization error and the focal width of the significant voxels obtained by localizing the dipoles placed at each depth. This was executed using the distributed dipolar method only. All simulated signals were added to the empirical baseline to retain the physiological SNR for the Gaussian pulses and the physiological power spectrum for the sinusoidal. The signals simulated were phase-locked (sinusoidal) and no time lags were added (Gaussian)

\section{Performance evaluation with various correlation}

Correlation in the data covariance is an important variable which can influence localizing capabilities of beamformers (Belardinelli et al., 2012). Therefore, we simulated multiple signals using the distributed dipolar model, consequently adding with the empirical baseline, such that there are various phase lags between the signal simulated in the left hemisphere and the right hemisphere. Four phase lags chosen for frequency domain analysis were $0, \pi / 2, \pi, 3 \pi / 2$. The power ratios were matched as per the acquired empirical power ratios. Four time lags chosen for time domain analysis were: $0,15,30,45 \mathrm{~ms}$.

\section{FP percentage}

We compared the "sensitivity" and "specificity" of eLORETA, LCMV, and DICS, using ROC analysis (Metz, 1978). Here, we calculated the probability of incorrectly detecting an activation, also called FP. Ideal detection should suppress FP. After thresholding the z-scores, we identified the number of significant clusters in each hemisphere, visually. Further we ran k-means clustering over significant voxel locations in each hemisphere and identified the nearest cluster to the true dipole location. Defining the nearest significant cluster/s from the dipolar location/s as the true positive/s, we further defined the FP percentage by computing the ratio of number of significant voxels not present in the true positive (or nearest cluster) and the total number of significant voxels. We also compare the performance of all meth- 
ods under parametric variation of SNR at the source level and different kinds of source configurations, e.g., single-point dipole, two-point dipoles, and distributed dipoles.

\section{Hit rate}

To evaluate the accuracy of localizing algorithms, we computed the hit rate or true positives for each method. After thresholding the z-scores and obtaining the significant clusters (using k-means), we identified the number of significant source points in the nearest cluster within a distance of $15 \mathrm{~mm}$ from the simulated dipole location. These source points were defined as hits. The hit rate corresponds to the ratio of number of hits and total number of significant source points in the nearest cluster. Hit rates were calculated across all SNRs and power ratios, including lags and different phase differences, respectively, for single-point, two-point, and distributed dipoles.

\section{Code accessibility}

All codes used for simulation of data and source localization algorithms is available at the following GitHub repository https://github.com/arpan-toolboxes/QuantitativeSourcelmaging. The reader is encouraged to contact the authors in case of implementation issues.

\section{Empirical EEG recordings \\ Participants}

Ten healthy volunteers (eight males, two females) aged between 22 and 39 years (mean 28 years old) participated in the study after giving informed consent, following the guidelines approved by Institutional Human Ethics Board at National Brain Research Center. All participants were self-declared normal individuals with no history of hearing impairments and had either correct or corrected-tonormal vision and no history of neurologic disorders.

\section{Stimuli}

Volunteers had to remain stationary in a seated position within a sound-proof room and hear auditory stimuli through $10-\Omega$ insert earphones with disposable foam eartips, binaurally for $200 \mathrm{~s}$ while fixating at a visual cross. Additionally, they had a baseline block where they fixated at the visual cross for $200 \mathrm{~s}$ without any sounds being played. Sounds were pure tones of $1000-\mathrm{Hz}$ frequency and $25-\mathrm{ms}$ time duration, with $5 \%$ rise and fall times and were repeated with a frequency of $40 \mathrm{~Hz}$ during an ON block of 1-s duration interspersed between two OFF blocks where no auditory stimuli were presented. Stimuli were made using in STIM2 stimulus presentation system with audio box P/N 1105 at $85 \mathrm{~dB}$.

\section{Data collection and pre-processing}

EEG data were acquired in an acoustically shielded room with 64 channels NeuroScan (SynAmps2) system with 1-kHz sampling rate. Brain Products abrasive electrolyte gel (EASYCAP) was used to make contact with scalp surface and the impedance was maintained at values less than $5 \mathrm{k} \Omega$ for all volunteers. Baseline EEG data were recorded for $200 \mathrm{~s}$ with eyes open, no tone, and a fixation cross on a monitor in front of the participants. Baseline and binaural stimuli were presented while par- ticipants were asked to maintain fixation on the cross all along to reduce eye movements.

Recorded raw data were re-referenced with average reference and were detrended to remove linear trends from the signal. Epochs of 5-s duration were constructed by concatenating ON blocks of $1 \mathrm{~s}$ each after removal of an initial $50 \mathrm{~s}$ of the 200-s-long session. This was done to capture ASSRs. Data were band pass filtered with cutoff frequencies $5-48 \mathrm{~Hz}$, to concentrate on sources underlying ASSR.

For an evoked wave form analysis, after average rereferencing, epochs of 1-s duration of ON blocks were extracted from the raw data during stimulus condition, then filtered with cutoff frequencies $0.5-48 \mathrm{~Hz}$, detrended, and averaged across trials to generate the evoked potential. Thresholds of -100 and $100 \mu \mathrm{V}$ were used to reject blinkcorrupted trials, meaning if at any point within the epoch the voltage exceeded the threshold values, the entire trial was deleted from the subsequent analysis.

Sensor locations were taken from the template given in the fieldtrip toolbox. Colin 27 structural T1 was used for co-registration with the sensor locations for accurate source localization. A forward model was computed using boundary element method (BEM) from the respective $\mathrm{T} 1$ image. For localization using the algorithms, we considered $0 \%$ regularization for all methods. The ratio of source power between stimulus and baseline condition was calculated in each voxel, using (Power(Stimulus)-Power(Baseline))./Power(Baseline) for the current density measures and (Power(Stimulus)-Power(Baseline)) for the beamformers. After computing the source intensities in each volunteer, the individual grids were interpolated to the T1 image. The averaged voxel intensities across all participants were evaluated using non-parametric statistics, and z-scores were computed for each hemisphere. The top $0.05 \%$ voxels were identified as sources.

\section{Results}

\section{Simulated EEG data}

Simulated EEG data were computed by placing electric dipolar sources at auditory cortical locations, according to single dipole, two-point dipoles and distributed dipole configurations, using Equation 1 and projecting the source activity at realistic sensor locations of a Neuroscan (Compumedics Inc) EEG cap using a realistic head model (Equation 2; Baillet et al., 2001). We considered two types of temporal profiles for source activity, a sinusoidal signal mimicking the band-specific frequency response observed in typical EEG signal such as ASSR and a mixture of Gaussian pulses representing the time-locked ERPs. Baseline data were acquired empirically on which both the aforementioned signals were added. Two prototype examples of simulated scalp activity during task and baseline are illustrated in Figure 1. To observe the effects of correlation in the CSD matrix on source localization, phase lags were introduced to the simulated sinusoidal signals generated from each hemisphere (two-point and distributed dipoles), as well as, time delay was added to the Gaussian pulses to the signal from the right hemisphere in two-point and distributed models. 
A

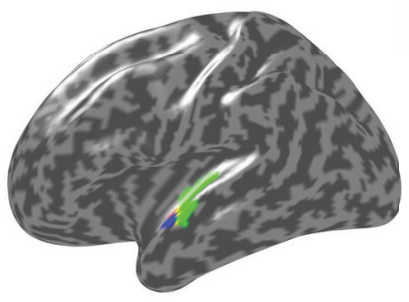

B

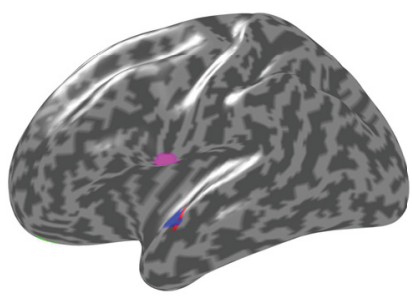

Left

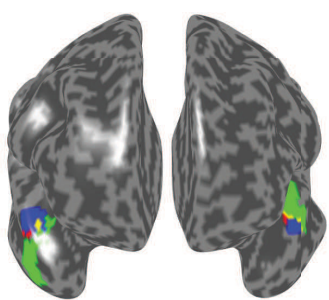

Simulated Dipole Position

eLORETA

DICS

Overlap: eLORETA and DICS

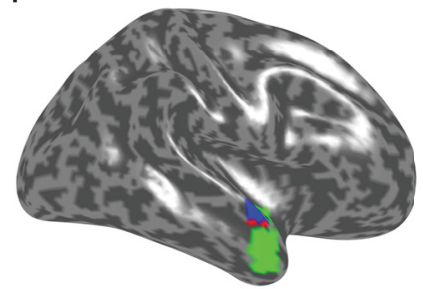

Simulated Dipole Position

eLORETA

LCMV

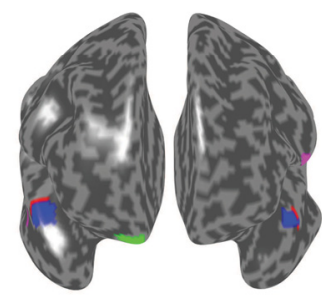

Front

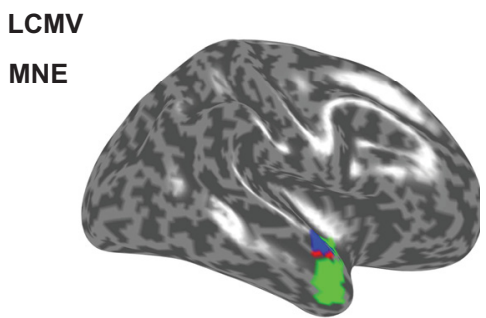

Figure 2. A, eLORETA (red) versus DICS source localization (green) using frequency-lock analyses on distributed dipolar source generated signals. Overlapping regions from both analyses are depicted in yellow and locations used for simulated dipole placements are depicted in blue. eLORETA was applied to the simulated sinusoidal signal embedded in resting state EEG, where the simulated signals generated from each hemisphere had 0 phase lag. For DICS, the simulated signal from the right hemisphere had a phase lag of $\pi$ with respect to the signal from the left hemisphere. The ratio of power spectrum at $40 \mathrm{~Hz}$ between the sinusoidal embedded in EEG and resting state EEG was chosen similar to the ratio found in our empirical results. $\boldsymbol{B}$, eLORETA (red), LCMV (green), and MNE (purple) generated sources using time-lock analysis. No overlapping regions were found. The results indicate 0 time-lag scenario between the Gaussian pulses from each hemisphere. Physiologically realistic SNR 25 dB for simulated dipolar sources was chosen for this illustration.

We applied eLORETA and DICS to perform frequencylocked source analysis on the sinusoidal data. Keeping the empirical baseline intact, we scaled the sinusoidal signal such that, we obtained different ratios of power of the sinusoidal at $40 \mathrm{~Hz}$, comparable to the values we found in our empirical data. Figure $2 A$, illustrates combined results from eLORETA and DICS algorithms on a brain surface rendered by the MNI brain, at power ratio of 1 (realistic SNR), for the distributed dipole model.

The algorithms were employed for localizing the sources of the peak negative response in mixture of Gaussians signal, by selecting a time segment constituting of points $\pm 25 \mathrm{~ms}$ around the peak (Fig. 1B). For plotting activations, the source locations in the $3 \mathrm{D}$ voxel space was projected to a surface plot using customized MATLAB codes.

The localization error was computed by first defining each voxel as point in a cluster and thereby determining the distance of the voxel with the maximum z-score from the true dipole source in each hemisphere. For distributed dipolar sources, the center of the spherically distributed source was considered as the true dipole location. The average of sum of distances from all such points to the voxel with the maximum z-score, normalized by the total number of points was used to quantify the focal localization of sources, in each hemisphere. All voxels were then transformed to their nearest projections on the cortical surface and identified as possible source locations in Figure 2. The quantitative evaluation of the performances of the inverse methods are addressed as follows.

\section{Accuracy}

Frequency analyses using eLORETA and DICS yielded similar localization errors with respect to different power ratios, in one dipole condition giving 0 FPs (Fig. 3). However, eLORETA provided much lower localization error than DICS for two-point and distributed dipole conditions. This was observed at lags: $0, \pi / 2$, and $3 \pi / 2$, where DICS performed comparatively poorly. Interestingly, DICS performed better than eLORETA at phase lag of $\pi$ in terms of accuracy, at which eLORETA's accuracy deteriorated. Overall, the most significant observation was linked to the 
Localization Error

A
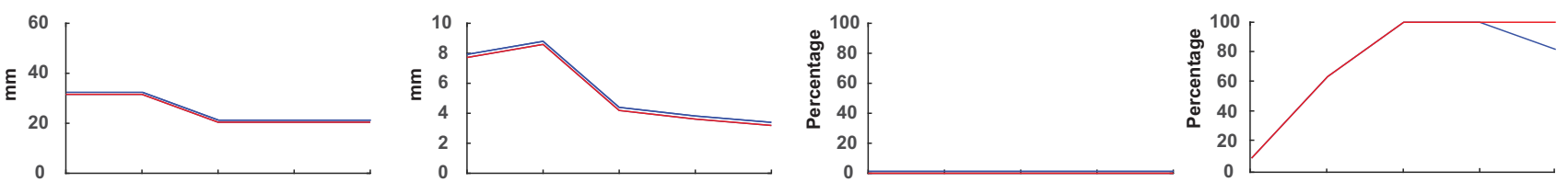

B
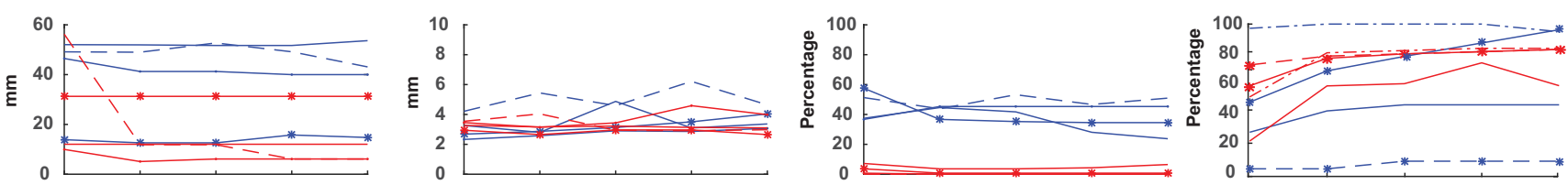

C
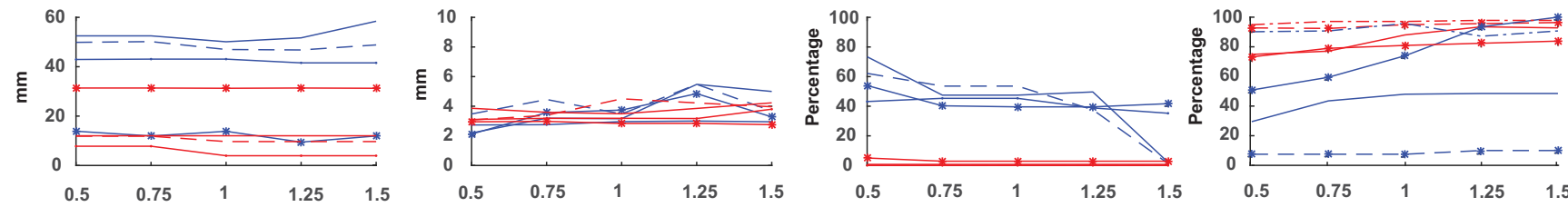

Ratio of simulated and empirical power spectrum at $40 \mathrm{~Hz}$
False Positives
Focal Width
eLORETA

DICS

Hit Rate

- 0 phase $\quad-\quad \pi / 2 \quad$ - $^{*} \pi \quad-3 \pi / 2$

Figure 3. Localization error, focal width, FP, and hit rate percentage for $(\boldsymbol{A})$ single dipole, (B) two-point dipole, and $(\boldsymbol{C})$ distributed dipole conditions measured for eLORETA (red) and DICS (blue). Source localization was done for all power ratios ( $x$-axis) across different simulated phase lags of $0, \pi / 2, \pi$, and $3 \pi / 2$.

consistent performance of the algorithms regardless of the power spectra at $40 \mathrm{~Hz}$.

In time domain analysis, eLORETA performed better in all dipole configurations in contrast to MNE and LCMV (Fig. 4). eLORETA consistently provided localization error of around $1 \mathrm{~cm}$ or less for all time lags. LCMV's accuracy with respect to SNR's and time lags was not consistent as no trend could be observed. In contrast, MNE was observed to be better than LCMV at almost all scenarios with consistent errors across all SNRs. Therefore, it can be pointed that SNR and time lags affect the performance of the beamformers and minimal effects can be found in the current density measures, in terms of accuracy.

\section{Localization spread}

eLORETA performance is observed to be comparable (similar focal localization) to DICS across all dipole conditions as well as power ratios (Fig. 3). Apart from slightly higher values of focal localization for DICS at $\pi / 2$ lag, both algorithms are efficiently focal.

The focal localization of eLORETA on time-locked signal was observed to reduce for single- and two-dipole condition, across different SNRs, however, not varying across time lags (Fig. 4). MNE and LCMV performance was comparable and also did not vary across time lags. However, the focal width of eLORETA deteriorated in the distributed dipole condition and varied heavily across time lags, in contrast to unperturbed performance of LCMV and MNE. Additionally, the focal width of eLORETA increased with increasing SNR. However, it was noted that there was minimal effect of SNR on the focal width of the other algorithms.

\section{Depth}

To quantitatively measure the localizing capabilities of eLORETA, DICS, MNE, and LCMV, source localization was executed for distributed dipoles at different depths. The localization error and the focal width were computed for the significant voxels, illustrated in Figure 5. The depth of dipolar locations was varied along the $x$-axis according to the $\mathrm{MNI}$ template, $1 \mathrm{~mm}$ apart for each localization iteration. The deepest positions were selected as $[-40$, $-28,6]$ in the left hemisphere and $[40,-24,6]$ in the right hemisphere. The locations closest to the surface were chosen as $[-60,-28,6]$ in the left hemisphere and $[60,28$, 8] in the right hemisphere. 


\section{Localization Error}

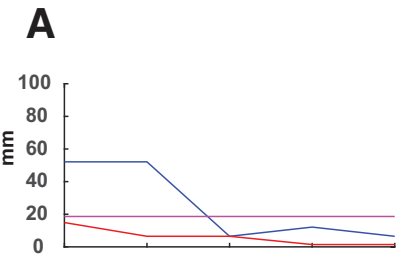

B
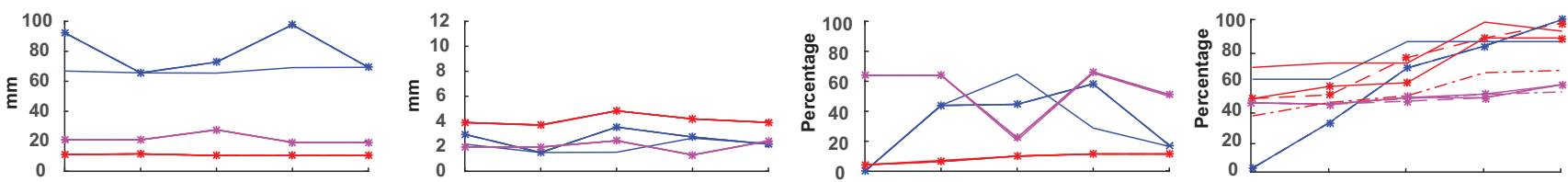

C
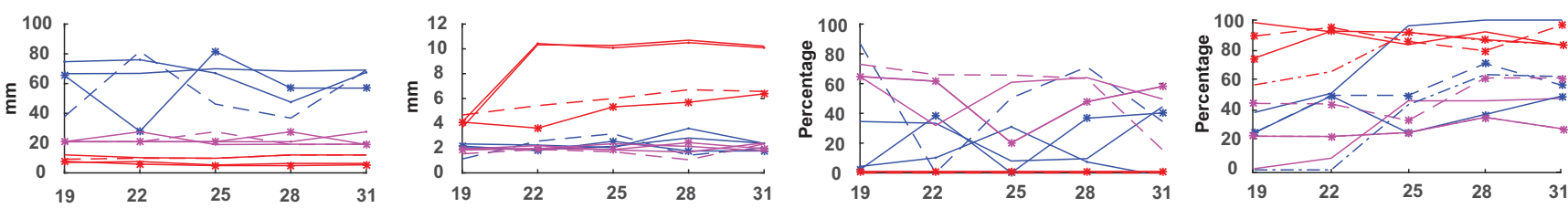

SNR

- $0 \mathrm{~ms} \mathrm{lag} \mathrm{\quad} \mathrm{-} 15 \mathrm{~ms} \mathrm{lag}$
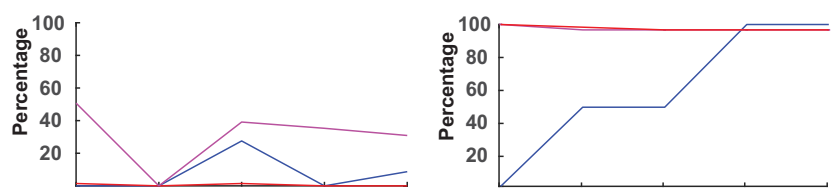

Hit Rate

eLORETA

LCMV

MNE

False Positives 
B

MNE
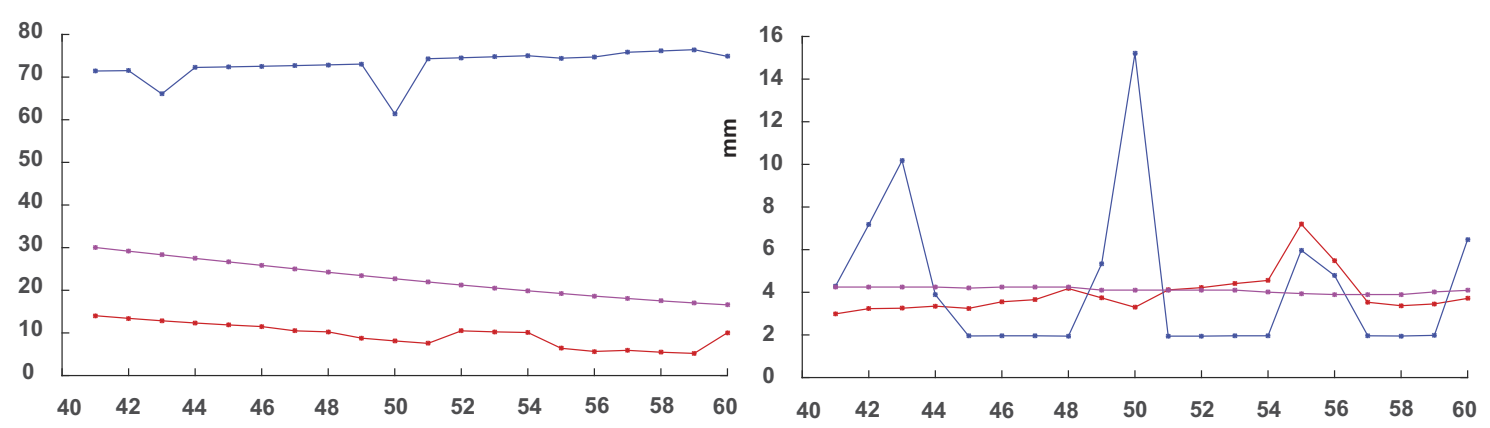

C

D

eLORETA

DICS

DICS

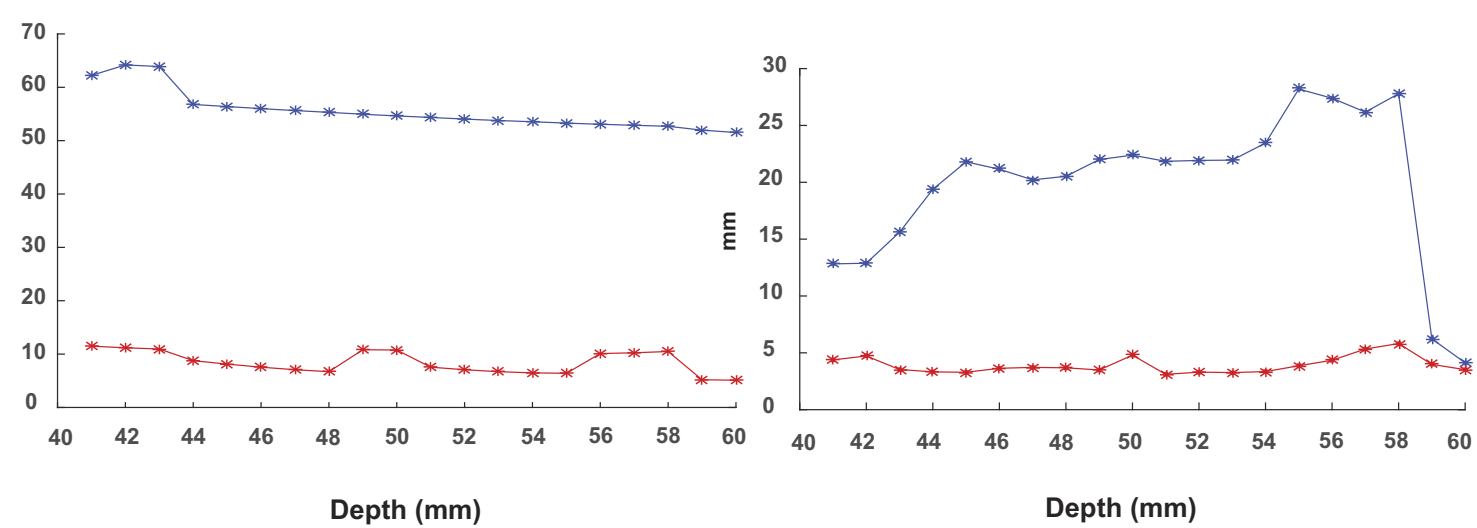

Figure 5. Localization errors and focal widths of all significant voxels computed for simulated distributed dipoles with the center of the dipole cluster located at different depths from the auditory cortical locations. The depth of the dipoles decreases along the $x$-axis; the distance from the auditory cortex. $\boldsymbol{A}, \boldsymbol{B}$, Localization error and the focal width of the sources generated by the mixture of Gaussian signals, respectively. $\boldsymbol{C}, \boldsymbol{D}$, exhibit the localization error and focal width for frequency domain analysis.

dipole conditions for localizing $40-\mathrm{Hz}$ sinusoidal signal (frequency analysis) and Gaussian pulse response (time lock), respectively.

\section{Hit rate}

eLORETA and DICS proved to be reliable in localizing single dipole, by yielding $100 \%$ hit rates at SNRs of biological level and higher. However, DICS indicated varied results with respect to the different power ratios at $40 \mathrm{~Hz}$ between the simulated and empirical data. In both, distributed and two-point dipolar model as the hit rates plummeted at phase difference of $\pi$. In contrast, eLORETA had high hit rates irrespective of phase differences and further increased with higher number of di- poles. For algorithms localizing the temporal features of the signal, the beamformer LCMV's performance was influenced by SNR, i.e., hit rates increased with increasing SNR's across all models. However, this was not true for the current density measures, since they were minimally influenced by SNR. Despite almost perfect localization of single dipole by current density measures, eLORETA yielded higher hit rates compared to MNE in two point and distributed dipole models. Similar to DICS, the hit rates corresponding to LCMV varied with respect to different temporal lags. To summarize, eLORETA's hit rates were superior among beamformers and current density measures. 
Table 1. Outcome of source localization performance based on different metrics for frequency analyses (eLORETA, DICS) and time domain analyses (eLORETA, LCMV, MNE)

\begin{tabular}{|c|c|c|c|}
\hline \multicolumn{4}{|c|}{ Frequency analysis } \\
\hline $\begin{array}{l}\text { Dipole condition } \\
\text { Single dipole }\end{array}$ & $\begin{array}{l}\text { Localization error } \\
\text { eLORETA/DICS }\end{array}$ & $\begin{array}{l}\text { Focal width } \\
\text { eLORETA/DICS }\end{array}$ & $\begin{array}{l}\text { False positives (FPs) } \\
\text { eLORETA/DICS }\end{array}$ \\
\hline Two-point dipoles & $\operatorname{eLORETA}(0, \pi / 2,3 \pi / 2) / \operatorname{DICS}(\pi)$ & eLORETA & eLORETA \\
\hline Single dipole & eLORETA & MNE/LCMV & eLORETA \\
\hline Two-point dipoles & eLORETA & MNE/LCMV & eLORETA \\
\hline
\end{tabular}

If similar performance was achieved, both methods are mentioned with "/."

\section{Empirical EEG data}

Source localization underlying 40-Hz EEG activity

The Fourier spectrum of each EEG channel time series was computed by multi-taper method with number of tapers $=2$, using Fieldtrip function ft_freqanalysis.m.
Power spectral density of empirical EEG data and the ERP time locked to the onset of a single tone stimulus are shown in Figure 6. In Figure $6 A$, the topoplot of the difference in power between binaural and baseline conditions at $40 \mathrm{~Hz}$ is shown along with the log of power

\section{A}
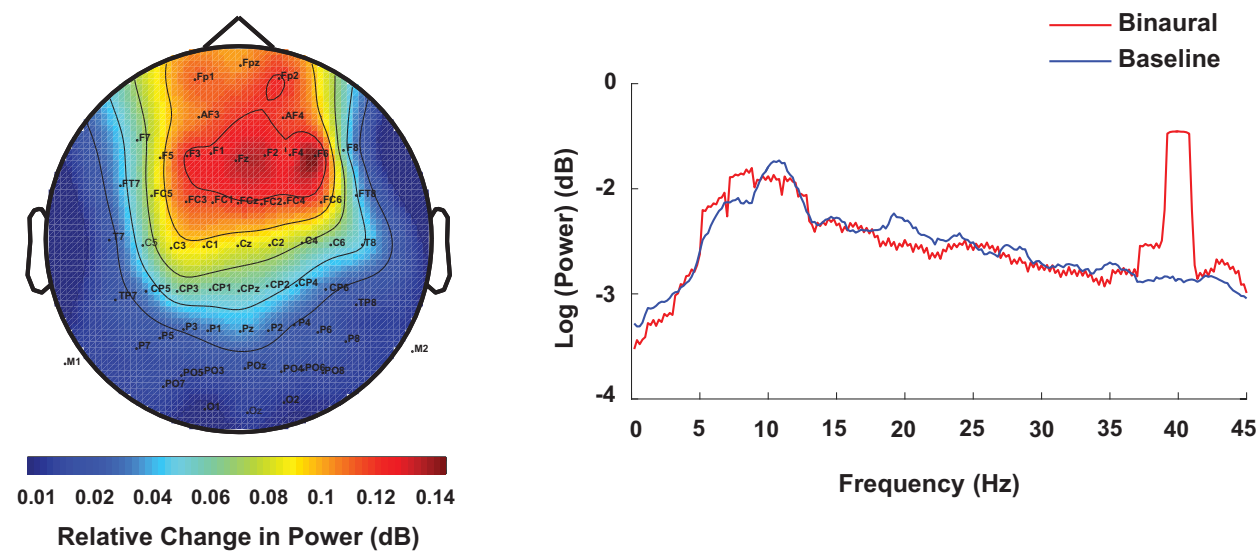

B

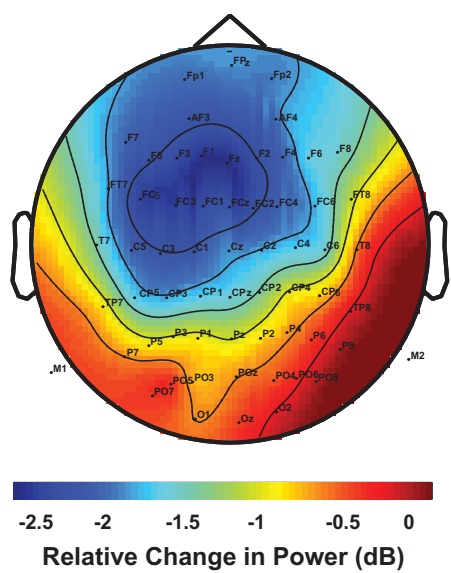

Binaural at M1 $\longrightarrow$ and $\mathrm{M} 2$

Baseline at M1

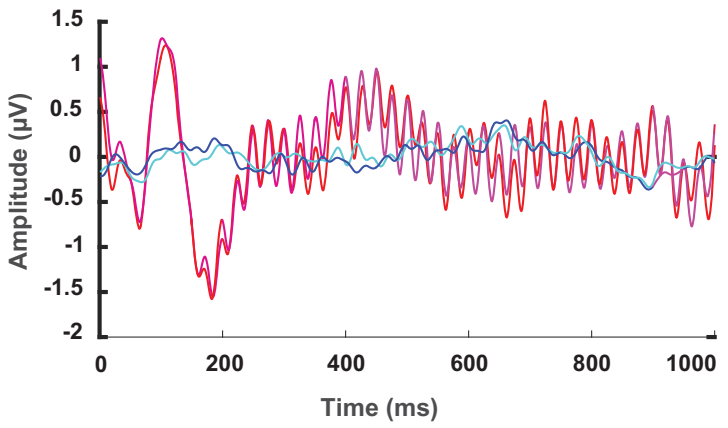

Figure 6. A, Topoplot of spectral power difference at $40 \mathrm{~Hz}$ and grand average of spectral power across all sensors, trials, and participants in binaural and the baseline conditions. Power spectral density was calculated for 5 -s windows after rejecting an initial $50 \mathrm{~s}$ out of total duration of $200 \mathrm{~s}$ for which the rhythmic tones were played. B, ERP responses of channels M1 and M2 across trials and participants for binaural and silent baseline conditions and the topoplot for the difference signal at the peak of N100 response (at $110 \mathrm{~ms}$ ). 
A
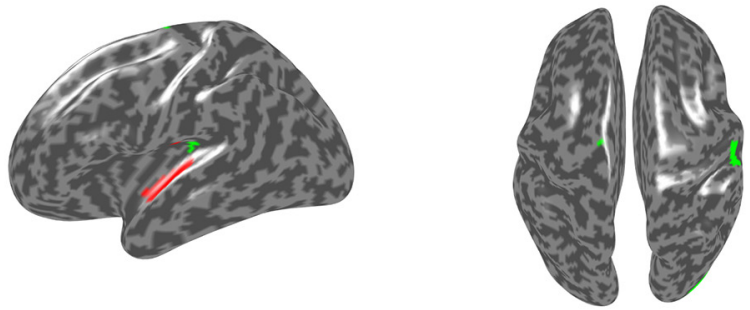

eLORETA

DICS

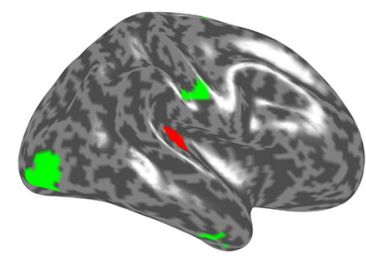

B

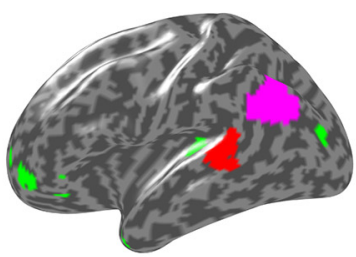

Left

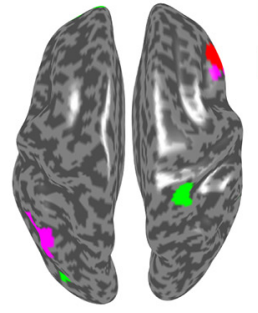

Top
eLORETA

LCMV

MNE

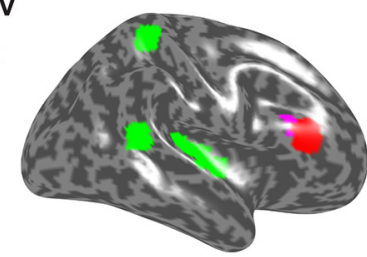

Figure 7. Left, Top and right view of significantly active cortical sources underlying for $(\boldsymbol{A}) 40-\mathrm{Hz}$ ASSR and (B) N100 component of the ERP. The red colored regions show estimated sources from eLORETA while green regions show estimated sources from $(\boldsymbol{A})$ DICS and $(\boldsymbol{B})$ LCMV analysis. The pink regions in $\boldsymbol{B}$ show MNE source localization results.

across all trials. There are peaks at alpha band $8-12 \mathrm{~Hz}$ in both binaural and baseline conditions the difference between which was not significant (two-sample $t=-0.018$, $p=0.95)$, whereas the binaural condition had a sharp rise in power at $40 \mathrm{~Hz}$, which was significant (two-sample $t=$ $0.18, p<0.0001$ ). The $t$ tests were performed on logarithm of power spectrums from two conditions within a frequency bands, $8-12 \mathrm{~Hz}$ for alpha and $39.8-40.2 \mathrm{~Hz}$ for evoked $40 \mathrm{~Hz}$. Localization results are illustrated in Figure $7 A$. The left, top, and right views of activation are shown where red regions represent sources from eLORETA analysis while green regions represent activation plots generated by DICS.

Acknowledging the absence of ground truth concerning true sources that exist in empirical data, we evaluated the focal width of all the significant clusters after thresholding the z-scores. One such cluster was found in each hemisphere around the auditory cortex for eLORETA, providing a mean focal width of $3.958 \mathrm{~mm}$. In contrast, DICS yielded one cluster at the auditory cortex in the left hemisphere and seven distributed clusters in the right. The mean focal width across DICS clusters was $2.8324 \mathrm{~mm}$, lesser than that of eLORETA. Understanding that the thresholding can vary focal width and number of clusters, we maintained the same threshold for both the algorithms for a fair comparison. It is to be noted that the mean focal width of two clusters of eLORETA can be decreased with higher thresholding.

\section{Source localization of N100 response}

In Figure 6B, we show the ERP responses to the binaural tone and the ERPs in baseline condition, averaged across all trials and participants (grand average). A negative peak around $100 \mathrm{~ms}$ after onset of tone stimulus (N100) was observed in the binaural condition with a latency of around $110 \mathrm{~ms}$. The topoplot represents the spatial map of the difference in relative changes of amplitude between ERPs from the binaural and baseline conditions across all channels and trials.

Next, we computed the underlying source activation during the N100 response using LCMV and eLORETA. In Figure $7 B$, we plot the source activations (top $0.05 \%$ voxels similar to $40-\mathrm{Hz}$ case) in epochs of duration $50 \mathrm{~ms}$, within which the 25th millisecond corresponds to the peak of N100. The beamformer localized bilateral auditory cortices, along with other distributed significant clusters. It can be noted that LCMV may localize the underlying activity, however, has higher probability of yielding FPs, due to its distributed activations. In contrast, the current density measures localized the left auditory cortex (eLORETA) and left posterior superior temporal sulcus (MNE). Computing only one cluster for each hemisphere, eLORETA and MNE yielded a significant cluster in the right frontal regions. The focal width of LCMV $(2.1706 \mathrm{~mm})$ was lesser than current density measures $(3.1-3.3 \mathrm{~mm})$, similar to the case in the frequency domain analysis. 


\section{Discussion}

Identifying the sources underlying key events of information processing such as ERP peaks or oscillatory brain activity such as spontaneous gamma oscillations are the objectives of many research studies. However, different inverse methods provide different solutions leading to no agreement of which algorithm is the "best method," as we illustrated in this manuscript with simulated and empirical EEG data. Although the selection of the best method can be guided by the nature of the hypothesis in a putative experimental design, a systematic comparative account of the efficacies of few prominent methods is currently missing in the literature. To address this issue we compared methods, eLORETA (Pascual-Marqui, 2007), LCMV (Van Veen et al., 1997), MNE (Hämäläinen and IImoniemi, 1994), and DICS (Gross et al., 2001) using the metrics that evaluates accuracy, sensitivity, and specificity across three dipolar models (single, two-point, and distributed) for the simulated sinusoidal signal (mimicking the steady state $40 \mathrm{~Hz}$ ) and a mixture of Gaussian pulses (representing the time locked ERP). Furthermore, we chose an empirically observed baseline which is a key ingredient for every inverse method. The models were simplistic, however, since we knew the exact location of dipole/s, ground truth validation was possible. All methods are able to retrieve the location of the true dipolar sources for a physiologically relevant SNR and frequency power ratio (Fig. 2, blue areas). Nonetheless, the study was conducted across different SNRs and frequency power spectra to test each method's sensitivity and specificity to noise. Furthermore, performance of all methods was evaluated with respect to dipolar depth, phase lags among sources, accuracy and localization spread over space. Also, we conducted source localization by collecting empirical EEG data exhibiting 40-Hz ASSR (Fig. 7). DICS and eLORETA were used to compute the sources underlying the $40-\mathrm{Hz}$ activity, and LCMV and eLORETA were used to compute the sources underlying the N100 response (Fig. 7). Thus, we could outlay the hallmarks of each method in an organized framework. The key finding of our study is that although high hit rates and focal localization are achieved with both current density and beamformer approaches, the FPs and focal width of sources needs to be carefully considered while choosing a specific method, and this is where eLORETA scores above most of the other methods.

A general consensus emerges from comparing the algorithms that there is no clear winner (Table 1) if accuracy as well as sensitivity and specificity are all taken together as guiding parameters. DICS gives better accuracy than eLORETA in single- and two-point dipole conditions even at low SNRs; however, the focal width of eLORETA generated sources is always slightly better most likely due to the minimization of the surface Laplacian component while estimation of the spatial filter. For distributed dipole scenario, focal width of eLORETA results were very similar to DICS, in fact getting better with higher SNR. Interestingly, eLORETA shows significant control on the FP ratio in the distributed dipole condition, proving to be the method of choice for estimating sources underlying fre- quency response in a more exploratory setting. This is indeed a very important point to note for increasing number of studies studying resting state functional connectivity (Canuet et al., 2011; Custo et al., 2017). Once a hypothesis is put in place with some prior knowledge about the involvement of prospective brain networks, one can go for DICS that can produce more accurate results (Tan et al., 2016). Interestingly, DICS results in lowest localization error with maximum phase lag of $\pi$, whereas the effect was reverse for eLORETA.

For source localization of ERP peaks, eLORETA majorly yielded better accuracy and specificity (in terms of favorable FPs) than LCMV and MNE, although the focal width of eLORETA sources were considerably larger than LCMV and MNE. The pitfalls and advantages of each method are summarized in Table 1. As in the case of frequency domain analysis, we would recommend eLORETA for an exploratory level analysis, whereas LCMV or MNE for more hypothesis-driven identification of sources.

An alternative solution to increase the probability of isolating an active source is to combine two or more methods and take the overlap of sources detected from them as the plausible source configuration. We provide a blueprint of these using our empirical and simulated data. Both spectral domain eLORETA and DICS were able to pinpoint the left auditory cortex, the location of one true source (Fig. 2). The number of FPs obtained with two methods combined is drastically low. This gives us the confidence that ASSR involves strong activity in primary sensory regions of auditory processing, e.g., bilateral middle temporal gyrus as reported by earlier studies (McFadden et al., 2014; Tan et al., 2016) and as we observe in Figure 7. However, for current empirical data, we did not find overlapping regions from different techniques, validation from two or more methods will give a strong confidence in the result of source localization. Proof-ofconcept illustration is available for time-lock analysis as well (Fig. 7), where we present combined eLORETA, LCMV, and MNE to identify the sources underlying N100 peak.

In future, we think an overlap-approach might result in focal localization with minimum number of FPs. This will particularly benefit the identification of sources whose activity may be relevant for a particular context. For example cross-frequency coupling (CFC) between alpha and gamma rhythms are being postulated to be important for gating of attention (Klimesch, 2012). How to identify a cortical subnetwork whose nodes show CFC out of the whole alpha and gamma networks is an important methodological challenge. We believe a conjunction of methods strategy to identify the potential sources will be crucial from the perspective of reliability as well as accuracy. In summary, our study provides a blue print for employing source-localization techniques to isolate more subtle features of signal processing.

\section{References}

Baillet S, Mosher JC, Leahy RM (2001) Electromagnetic brain mapping. IEEE Signal Proc Mag 18:14-30.

Barnes GR, Hillebrand A (2003) Statistical flattening of MEG beamformer images. Hum Brain Mapp 18:1-12 
Belardinelli P, Ortiz E, Barnes G, Noppeney U, Preissl H (2012) Source reconstruction accuracy of MEG and EEG Bayesian inversion approaches. PloS One 7:e51985.

Bollimunta A, Chen Y, Schroeder CE, Ding M (2008) Neuronal mechanisms of cortical alpha oscillations in awake-behaving macaques. J Neurosci 28:9976-9988.

Bradley A, Yao J, Dewald J, Richter C-P (2016) Evaluation of electroencephalography source localization algorithms with multiple cortical sources. PloS One 11:e0147266.

Bressler SL, Kelso JAS (2001) Cortical coordination dynamics and cognition. Trends Cogn Sci 5:26-36.

Bressler SL, Coppola R, Nakamura R (1993) Episodic multiregional cortical coherence at multiple frequencies during visual task performance. Nature 366:153-156.

Brovelli A, Ding M, Ledberg A, Chen Y, Nakamura R, Bressler SL (2004) Beta oscillations in a large-scale sensorimotor cortical network: directional influences revealed by Granger causality. Proc Natl Acad Sci USA 101:9849-9854.

Buzsáki G, Draguhn A (2004) Neuronal oscillations in cortical networks. Science 304:1926-1929.

Canuet L, Ishii R, Pascual-Marqui RD, Iwase M, Kurimoto R, Aoki Y, Ikeda S, Takahashi H, Nakahachi T, Takeda M (2011) Resting-state EEG source localization and functional connectivity in schizophrenia-like psychosis of epilepsy. PLoS One 6:e27863.

Cheyne D, Ferrari P (2013) MEG studies of motor cortex gamma oscillations: evidence for a gamma "fingerprint" in the brain? Front Hum Neurosci 7:575.

Cheyne D, Bakhtazad L, Gaetz W (2006) Spatiotemporal mapping of cortical activity accompanying voluntary movements using an event-related beamforming approach. Hum Brain Mapp 27:213229.

Clark VP, Fan S, Hillyard S (1994) Identification of early visual evoked potential generators by retinotopic and topographic analyses. Hum Brain Mapp 2:170-187.

Custo A, Van De Ville D, Wells WM, Tomescu MI, Brunet D, Michel CM (2017) Electroencephalographic resting-state networks: source localization of microstates. Brain Connect 7:671-682.

Edelman G, V, Mountcastle (1978). An organizing principle for cerebral function: the unit model and the distributed system. In: The mindful brain. Cambridge, MA: MIT Press.

Goldenholz DM, Ahlfors SP, Hämäläinen MS, Sharon D, Ishitobi M, Vaina LM, Stufflebeam SM (2009) Mapping the signal-to-noiseratios of cortical sources in magnetoencephalography and electroencephalography. Hum Brain Mapp 30:1077-1086.

Gross J, Kujala J, Hamalainen M, Timmermann L, Schnitzler A, Salmelin R (2001) Dynamic imaging of coherent sources: studying neural interactions in the human brain. Proc Natl Acad Sci USA 98:694-699.

Hämäläinen MS, Sarvas J (1989) Realistic conductivity geometry model of the human head for interpretation of neuromagnetic data. IEEE Trans Biomed Eng 36:165-171.

Hämäläinen MS, Ilmoniemi RJ (1994) Interpreting magnetic fields of the brain: minimum norm estimates. Med Biol Eng Comput 32:3542.

Hedrich T, Pellegrino G, Kobayashi E, Lina JM, Grova C (2017) Comparison of the spatial resolution of source imaging techniques in high-density EEG and MEG. Neuroimage 157:531-544.

Hillebrand A, Barnes GR (2002) A quantitative assessment of the sensitivity of whole-head MEG to activity in the adult human cortex. Neuroimage 16:638-650.

Hillebrand A, Barnes GR (2003) The use of anatomical constraints with MEG beamformers. Neuroimage 20:2302-2313.

Ishii $R$, Shinosaki $K$, Ukai $S$, Inouye $T$, Ishihara $T$, Yoshimine $T$, Hirabuki N, Asada H, Kihara T, Robinson SE, Takeda M (1999) Medial prefrontal cortex generates frontal midline theta rhythm. Neuroreport 10:675-679.

Klimesch W (2012) Alpha-band oscillations, attention, and controlled access to stored information. Trends Cogn Sci 16:606-617.
Kutas M, McCarthy G, Donchin E (1977) Augmenting mental chronometry: the p300 as a measure of stimulus evaluation time. Science 197:792-795.

Lina JM, Chowdhury R, Lemay E, Kobayashi E, Grova C (2014) Wavelet-based localization of oscillatory sources from magnetoencephalography data. IEEE Trans Biomed Eng 61:2350-2364.

Liu AK, Dale AM, Belliveau JW (2002) Monte carlo simulation studies of eeg and meg localization accuracy. Hum Brain Mapp 16:47-62.

Llinás RR, Ribary U, Jeanmonod D, Kronberg E, Mitra PP (1999) Thalamocortical dysrhythmia: a neurological and neuropsychiatric syndrome characterized by magnetoencephalography. Proc Natl Acad Sci USA 96:15222-15227.

McFadden KL, Steinmetz SE, Carroll AM, Simon ST, Wallace A, Rojas DC (2014) Test-retest reliability of the $40 \mathrm{~Hz}$ EEG auditory steady-state response. PLoS One 9:e85748.

Metz CE (1978) Basic principles of roc analysis. Semin Nucl Med 8:283-298.

Mideksa KG, N, Hoogenboom H, Hellriegel H, Krause A, Schnitzler G, Deuschl J, Raethjen U, Heute, Muthuraman M (2015) Comparison of EEG and MEG in source localization of induced human gamma-band oscillations during visual stimulus. Proceedings of the Annual International Conference of the IEEE Engineering in Medicine and Biology Society 2015, 8119-8122.

Mosher JC, Leahy RM, Lewis PS (1999) EEG and MEG: forward solutions for inverse methods. IEEE Trans Biomed Eng 46:245-59.

Müller N, Weisz N (2012) Lateralized auditory cortical alpha band activity and interregional connectivity pattern reflect anticipation of target sounds. Cereb Cortex 22:1604-1613.

Murzin V, Fuchs A, Kelso JAS (2011) Anatomically constrained minimum variance beamforming applied to EEG. Exp Brain Res 214: 515-528.

Murzin V, Fuchs A, Kelso JAS (2013) Detection of correlated sources in eeg using combination of beamforming and surface laplacian methods. J Neurosci Methods 218:96-102.

Ou W, Hämäläinen MS, Golland P (2009) A distributed spatiotemporal EEG/MEG inverse solver. Neuroimage 44:932-946.

Pantev C, Bertrand O, Eulitz C, Verkindt C, Hampson S, Schuierer G, Elbert T (1995) Specific tonotopic organizations of different areas of the human auditory cortex revealed by simultaneous magnetic and electric recordings. Electroencephalogr Clin Neurophysiol 94: 26-40.

Pascual-Marqui RD (2007) Discrete, 3D distributed, linear imaging methods of electric neuronal activity. Part 1: exact, zero error localization. ArXiv 0710.3341. Available at http://arxiv.org/pdf/ 0710.3341.

Picton TW, Hillyard SA, Krausz HI, Galambos R (1974) Human auditory evoked potentials. I. Evaluation of component. Electroencephalogr Clin Neurophysiol 36:179-190.

Ramírez RR, Wipf D, Baillet S (2010) Neuroelectromagnetic source imaging of brain dynamics, pp 127-155. New York, NY: Springer.

Sato M, Yoshioka T, Kajihara S, Toyama K, Goda N, Doya K, Kawato M (2004) Hierarchical Bayesian estimation for MEG inverse problem. Neuroimage 23:806-826.

Srinivasan R, Winter WR, Nunez PL (2006) Source analysis of EEG oscillations using high-resolution EEG and MEG. Prog Brain Res 159:29-42.

Tan HM, Gross J, Uhlhaas PJ (2016) MEG sensor and source measures of visually induced gamma-band oscillations are highly reliable. Neuroimage 137:34-44.

Van Veen BD, van Drongelen W, Yuchtman M, Suzuki A (1997) Localization of brain electrical activity via linearly constrained minimum variance spatial filtering. IEEE Trans Biomed Eng 44:867880.

Varela F, Lachaux JP, Rodriguez E, Martinerie J (2001) The brainweb: phase synchronization and large-scale integration. Nat Rev Neurosci 2:229-239. 